\title{
Emission factors for gaseous and particulate pollutants from offshore diesel engine vessels in China
}

\section{Fan Zhang et al.}

Correspondence to: Yingjun Chen (yjchentj@tongji.edu.cn) and Chongguo Tian (cgtian@yic.ac.cn)

The copyright of individual parts of the supplement might differ from the CC-BY 3.0 licence. 
1 Real-world Measurement System for vessels

2 Detailed composition and procedure of the on-board measurement system were

3 given as follows: The whole measurement system was placed on deck next to the

4 exhaust pipe of the vessel. A slender tube was placed into the vessel exhaust pipe to

5 lead out the flue gas. Then it was divided into five subsamples through a manifold

6 for different analyses and evacuation of the excess gas. The on-board test picture

7 (Figure S1, a)) and schematic diagram of the portable measurement system (Figure

$8 \quad \mathrm{~S} 1, \mathrm{~b}))$ are shown in Figure $\mathrm{S} 1$.

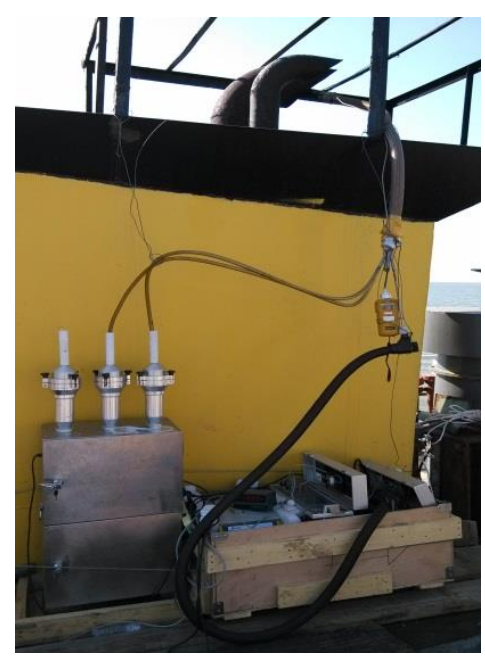

a)

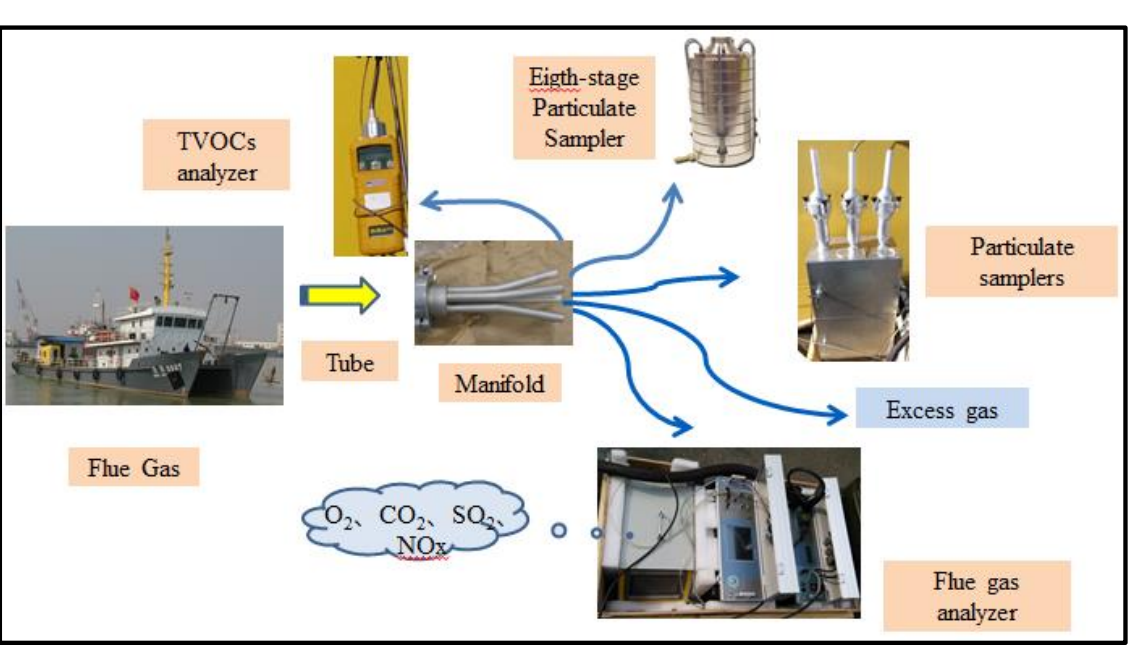

b)
9

10

Figure S1 Real-world measurement system for vessels: a) on-board test picture, b) schematic diagram of the portable measurement system 

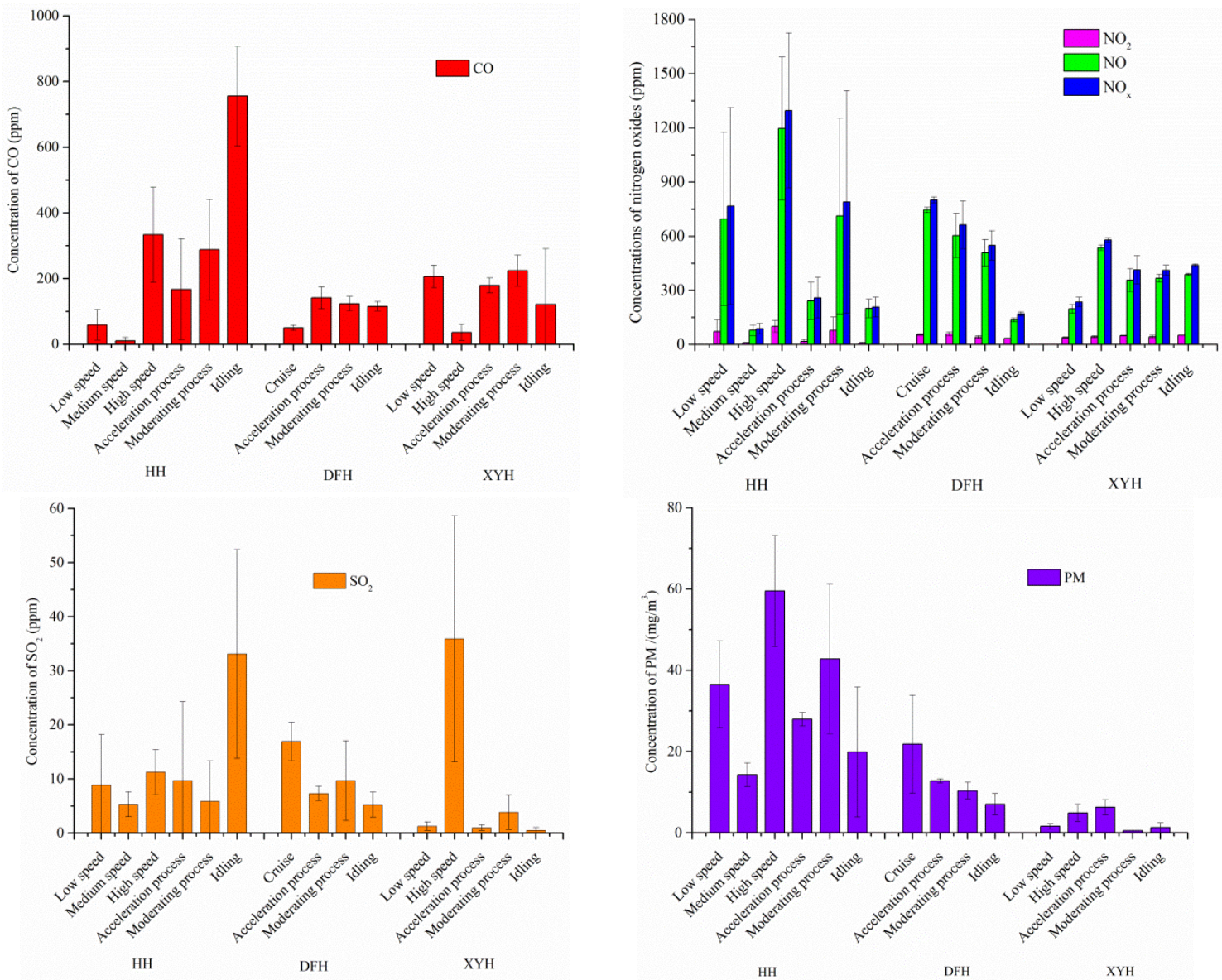

1

2 Figure $\mathrm{S} 2$. Concentrations of main gaseous matter and PM in shipping emissions 
Table S1 Types composition of offshore vessels in China

\begin{tabular}{lcc}
\hline & Vessel type & Percentage \% \\
\hline & Tanker & 18.03 \\
& Liquefied gas carrier & 1.11 \\
Cargo ship & Chemical tanker & 7.71 \\
& Bulk carrier & 14.98 \\
& Container ship & 5.61 \\
& Ro-ro ship & 3.21 \\
& Other cargo ships & 37.04 \\
\hline \multirow{2}{*}{ Other ship } & push-tow ship & 8.07 \\
& Barge & 1.07 \\
& Non-transport Ship & 3.17 \\
\hline
\end{tabular}

2

3 Table S2 Distribution of vessels through gross tonnage in 2014 in offshore area of

$4 \quad$ Yangtze River Delta

\begin{tabular}{cc}
\hline Gross tonnage $(\mathrm{t})$ & Percentage $(\%)$ \\
\hline $10000-49999$ & 3.7 \\
$3000-9999$ & 12.7 \\
$1000-2999$ & 50.3 \\
$500-999$ & 13.8 \\
$100-499$ & 8.2 \\
$<99$ & 7.0 \\
\hline
\end{tabular}

5

6 
1 Table S3 The Chinese market consumption of marine oil in 2011

\begin{tabular}{|c|c|c|c|}
\hline \multirow{2}{*}{ Oil type } & \multirow{2}{*}{ Application of ship type } & \multicolumn{2}{|c|}{$\begin{array}{l}\text { Quantity demand } \\
\qquad\left(10^{4} \text { ton }\right)\end{array}$} \\
\hline & & $\begin{array}{l}\text { Domestic } \\
\text { trade oil }\end{array}$ & $\begin{array}{l}\text { Bonded } \\
\text { oil }\end{array}$ \\
\hline $\begin{array}{l}\text { Light fuel oil (density between } 0.86 \\
\text { to } 0.92 \text { ) or equals DMA/DMB/DMC }\end{array}$ & $\begin{array}{c}\text { Vessels with medium speed } \\
\text { diesel engine of rmp above } 300\end{array}$ & 400 & 125 \\
\hline 180\# fuel oil & Vessels with low speed diesel & 410 & 257 \\
\hline $\begin{array}{l}\text { 380\# fuel oil } \\
\text { other heavy fuels }(120 \# / 250 \# / \text { above } \\
500 \# \text { etc. })\end{array}$ & $\begin{array}{c}\text { engine of rmp below } 300 \\
\text { 120\# applicable to medium or } \\
\text { small fishing vessel; others } \\
\text { applicable to new large vessels }\end{array}$ & 120 & 632 \\
\hline
\end{tabular}

2 
1 Table S4 Detection parameters for the gaseous matter

\begin{tabular}{ccccccc}
\hline Component & Method & Range & Resolution & Accuracy & $\begin{array}{c}\text { Time } \\
\left(\mathrm{T}_{90}\right)\end{array}$ & Conformity \\
\hline $\mathrm{O}_{2}$ & $\begin{array}{c}\text { Electrochemical } \\
\text { sensor }\end{array}$ & $20.95 \%$ & $0.01 \%$ & $\pm 5 \%$ rel. & $45 \mathrm{~s}$ & $\begin{array}{c}\text { ISO 12039, } \\
\text { CTM-030 }\end{array}$ \\
$\mathrm{CO}_{2}$ & NDIR & $5 \%$ & $0.01 \%$ & $\pm 3 \%$ rel. & $45 \mathrm{~s}$ & $\begin{array}{c}\text { ISO 12039, } \\
\text { OTM-13 }\end{array}$ \\
$\mathrm{CH}_{4}$ & NDIR & $5 \%$ & $0.01 \%$ & $\pm 3 \%$ rel. & $45 \mathrm{~s}$ & \\
$\mathrm{NO}$ & NDIR & $1000 \mathrm{ppm}$ & $1 \mathrm{ppm}$ & $\pm 3 \%$ rel. & $45 \mathrm{~s}$ & $\begin{array}{c}\text { ISO 10849, } \\
\text { Method 7E }\end{array}$ \\
$\mathrm{NO}_{2}$ & NDIR & $1000 \mathrm{ppm}$ & $1 \mathrm{ppm}$ & $\pm 3 \%$ rel. & $45 \mathrm{~s}$ & $\begin{array}{c}\text { ISO 10849, } \\
\text { Method 7E }\end{array}$ \\
$\mathrm{SO}_{2}$ & NDIR & $1000 \mathrm{ppm}$ & $1 \mathrm{ppm}$ & $\pm 3 \%$ rel. & $45 \mathrm{~s}$ & $\begin{array}{c}\text { ISO 7935, } \\
\text { Method 6C }\end{array}$ \\
$\mathrm{N}_{2} \mathrm{O}$ & NDIR & $2000 \mathrm{ppm}$ & $1 \mathrm{ppm}$ & $\pm 3 \%$ rel. & $45 \mathrm{~s}$ & ISO 21258 \\
$\mathrm{VOCl}^{\mathrm{NO}}$ & PID & $10000 \mathrm{ppm}$ & $0.1 \mathrm{ppm}$ & $\pm 5 \%$ rel. & - & \\
\hline
\end{tabular}

2 NDIR, Non-dispersive Infra-red

3 PID, Photo Ionization Detector 
1 Table S5. Fuel-based EFs for the gaseous pollutants for different operating modes

$2 \quad\left(\mathrm{~g} \cdot \mathrm{kg}^{-1}\right.$ fuel $)$

\begin{tabular}{|c|c|c|c|c|c|c|c|c|c|}
\hline $\begin{array}{c}\text { Vessel } \\
\text { ID }\end{array}$ & Operating mode & $\mathrm{CO}_{2}$ & $\mathrm{CO}$ & NO & $\mathrm{NO}_{2}$ & $\mathrm{~N}_{2} \mathrm{O}$ & $\mathrm{NO}_{\mathrm{X}}$ & TVOC & $\mathrm{SO}_{2}$ \\
\hline \multirow{6}{*}{$\mathrm{HH}$} & Low speed & $3023 \pm 2236$ & $6.08 \pm 4.77$ & $76.4 \pm 52.8$ & $12.0 \pm 11.2$ & $0.81 \pm 0.47$ & $89.2 \pm 64.3$ & $50.7 \pm 83.7$ & \multirow{6}{*}{1.60} \\
\hline & Medium speed & $3106 \pm 1753$ & $15.3 \pm 15.6$ & $121.6 \pm 43.9$ & $20.2 \pm 6.01$ & $0.77 \pm 1.69$ & $142.6 \pm 51.6$ & $20.6 \pm 19.4$ & \\
\hline & High speed & $3069 \pm 1342$ & $12.7 \pm 5.50$ & $48.6 \pm 16.1$ & $6.23 \pm 2.08$ & $1.82 \pm 0.34$ & $56.6 \pm 18.6$ & $29.9 \pm 23.6$ & \\
\hline & $\begin{array}{c}\text { Acceleration } \\
\text { process }\end{array}$ & $2940 \pm 1133$ & $45.2 \pm 41.7$ & $69.7 \pm 30.0$ & $7.87 \pm 4.59$ & $4.87 \pm 2.83$ & $82.5 \pm 37.4$ & $62.0 \pm 2.83$ & \\
\hline & $\begin{array}{l}\text { Moderating } \\
\text { process }\end{array}$ & $3049 \pm 2005$ & $26.0 \pm 13.8$ & $68.7 \pm 52.3$ & $11.5 \pm 11.2$ & $2.65 \pm 1.54$ & $82.8 \pm 65.0$ & $30.8 \pm 24.6$ & \\
\hline & Idling & $2948 \pm 514$ & $122 \pm 24.5$ & $34.7 \pm 8.86$ & $1.95 \pm 1.43$ & $2.45 \pm 2.84$ & $39.1 \pm 13.1$ & $14.1 \pm 8.04$ & \\
\hline \multirow{4}{*}{ DFH } & Cruise & $3160 \pm 141$ & $1.88 \pm 0.30$ & $29.9 \pm 0.59$ & $3.37 \pm 0.30$ & $0.01 \pm 0.01$ & $33.3 \pm 0.90$ & $1.24 \pm 0.07$ & \multirow{4}{*}{0.92} \\
\hline & $\begin{array}{c}\text { Acceleration } \\
\text { process }\end{array}$ & $3157 \pm 197$ & $5.91 \pm 1.38$ & $27.0 \pm 5.55$ & $4.06 \pm 0.62$ & $0.37 \pm 0.38$ & $31.5 \pm 6.55$ & - & \\
\hline & $\begin{array}{c}\text { Moderating } \\
\text { process }\end{array}$ & $3157 \pm 180$ & $5.81 \pm 1.02$ & $25.5 \pm 3.67$ & $3.15 \pm 0.61$ & $0.45 \pm 0.39$ & $29.1 \pm 4.68$ & - & \\
\hline & Idling & $3121 \pm 303$ & $28.2 \pm 3.59$ & $35.5 \pm 2.65$ & $13.5 \pm 0.72$ & $1.84 \pm 0.65$ & $50.8 \pm 4.02$ & - & \\
\hline \multirow{5}{*}{$\mathrm{XYH}$} & Low speed & $3102 \pm 409$ & $32.2 \pm 5.26$ & $32.9 \pm 4.04$ & $9.67 \pm 0.84$ & $0.65 \pm 0.42$ & $43.3 \pm 5.31$ & $9.15 \pm 0.00$ & \multirow{5}{*}{2.60} \\
\hline & High speed & $3162 \pm 75.7$ & $1.40 \pm 0.97$ & $22.4 \pm 0.63$ & $2.76 \pm 0.29$ & $0.28 \pm 0.12$ & $25.4 \pm 1.04$ & $4.41 \pm 0.00$ & \\
\hline & $\begin{array}{c}\text { Acceleration } \\
\text { process }\end{array}$ & $3150 \pm 732$ & $17.0 \pm 2.15$ & $36.2 \pm 6.49$ & $7.70 \pm 0.22$ & $0.15 \pm 0.06$ & $44.1 \pm 6.77$ & - & \\
\hline & $\begin{array}{c}\text { Moderating } \\
\text { process }\end{array}$ & $3148 \pm 166$ & $18.7 \pm 3.93$ & $32.8 \pm 1.94$ & $5.93 \pm 1.04$ & $0.05 \pm 0.07$ & $38.8 \pm 3.04$ & - & \\
\hline & Idling & $3162 \pm 72.4$ & $9.97 \pm 3.80$ & $34.0 \pm 0.56$ & $6.86 \pm 0.19$ & $0.05 \pm 0.02$ & $40.9 \pm 0.76$ & - & \\
\hline
\end{tabular}

$3-$-, Unavailable data for TVOC

4 
Table S6 Fuel-based EFs for PM and its chemical composition (PM, OC and EC are given as $\mathrm{g} \mathrm{kg}^{-1}$ fuel, others are given as mg kg $\mathrm{kuel}^{-1}$

\begin{tabular}{|c|c|c|c|c|c|c|c|c|c|c|c|c|c|c|c|}
\hline \multirow{2}{*}{$\begin{array}{c}\text { Vessel ID } \\
\text { Operating mode }\end{array}$} & \multicolumn{6}{|c|}{$\mathrm{HH}$} & \multicolumn{5}{|c|}{ DFH } & \multicolumn{3}{|c|}{$\mathrm{XYH}$} & \multirow[b]{2}{*}{ Idling } \\
\hline & Low speed & $\begin{array}{c}\text { Medium } \\
\text { speed }\end{array}$ & $\begin{array}{l}\text { High } \\
\text { speed }\end{array}$ & $\begin{array}{l}\text { Accelerati } \\
\text { on process }\end{array}$ & $\begin{array}{l}\text { i Moderating } \\
\text { s process }\end{array}$ & Idling & Cruise & $\begin{array}{l}\text { Acceleration } \\
\text { process }\end{array}$ & $\begin{array}{c}\text { Moderating } \\
\text { process }\end{array}$ & Idling & Low speed & High speed & $\begin{array}{l}\text { Accelerati } \\
\text { on process }\end{array}$ & $\begin{array}{c}\text { Moderating } \\
\text { process }\end{array}$ & \\
\hline $\mathrm{PM}$ & $1.29 \pm 0.52$ & $4.62 \pm 3.09$ & $1.00 \pm 0.49$ & $1.86 \pm 0.29$ & $0.97 \pm 0.30$ & $2.08 \pm 1.09$ & $0.65 \pm 0.36$ & $0.43 \pm 0.02$ & $0.39 \pm 0.08$ & $1.33 \pm 0.49$ & $0.19 \pm 0.08$ & $0.15 \pm 0.07$ & $0.47 \pm 0.14$ & $0.04 \pm 0.00$ & $0.09 \pm 0.08$ \\
\hline OC & $0.30 \pm 0.40$ & $0.82 \pm 0.31$ & $0.12 \pm 0.06$ & $60.14 \pm 0.02$ & $0.11 \pm 0.02$ & $0.51 \pm 0.34$ & $0.18 \pm 0.20$ & $0.09 \pm 0.03$ & $0.14 \pm 0.02$ & $0.56 \pm 0.14$ & $0.17 \pm 0.14$ & $0.05 \pm 0.02$ & $0.15 \pm 0.07$ & $0.05 \pm 0.02$ & $0.04 \pm 0.00$ \\
\hline EC & $0.47 \pm 0.20$ & $0.72 \pm 0.60$ & $0.58 \pm 0.25$ & $50.10 \pm 0.34$ & $0.15 \pm 0.00$ & $1.70 \pm 1.69$ & $0.22 \pm 0.16$ & $0.34 \pm 0.12$ & $0.19 \pm 0.03$ & $0.42 \pm 0.03$ & $0.04 \pm 0.02$ & $0.09 \pm 0.03$ & $0.22 \pm 0.12$ & $0.02 \pm 0.02$ & $0.03 \pm 0.00$ \\
\hline $\mathrm{NO}_{3}{ }^{-}$ & $13.4 \pm 2.72$ & ND & $3.40 \pm 1.99$ & ND & $10.5 \pm 4.66$ & $3.22 \pm 2.12$ & $17.8 \pm 26.8$ & $2.11 \pm 0.81$ & $0.64 \pm 0.54$ & $55.7 \pm 67.2$ & $2.27 \pm 0.51$ & $0.35 \pm 0.45$ & $2.95 \pm 3.89$ & $0.83 \pm 0.13$ & $8.38 \pm 0.00$ \\
\hline $\mathrm{SO}_{4}{ }^{2-}$ & $101 \pm 118$ & $52.2 \pm 20.0$ & $82.0 \pm 35.2$ & $11.5 \pm 8.19$ & $43.4 \pm 35.0$ & $24.9 \pm 9.62$ & $61.9 \pm 5.58$ & $28.2 \pm 4.72$ & $30.6 \pm 5.49$ & $8.87 \pm 0.00$ & $1.81 \pm 2.08$ & $21.6 \pm 5.47$ & $27.1 \pm 5.78$ & $12.4 \pm 0.00$ & $2.45 \pm 0.00$ \\
\hline $\mathrm{NH}_{4}^{+}$ & $16.0 \pm 8.01$ & $86.9 \pm 16.5$ & $7.76 \pm 1.45$ & $29.2 \pm 0.03$ & $20.9 \pm 9.41$ & $15.9 \pm 6.07$ & $5.27 \pm 4.52$ & $1.48 \pm 1.07$ & $3.11 \pm 0.78$ & $2.51 \pm 1.62$ & $2.85 \pm 0.20$ & $3.71 \pm 0.92$ & $3.01 \pm 1.72$ & $4.03 \pm 1.75$ & $4.97 \pm 0.00$ \\
\hline $\mathrm{Ca}^{2+}$ & $4.25 \pm 1.10$ & $23.7 \pm 10.3$ & $6.43 \pm 1.57$ & $12.1 \pm 6.77$ & $15.9 \pm 0.00$ & $17.3 \pm 0.46$ & $4.11 \pm 0.48$ & $4.61 \pm 2.33$ & $1.58 \pm 0.90$ & $3.48 \pm 0.00$ & $1.30 \pm 0.58$ & $1.62 \pm 1.31$ & $0.65 \pm 0.35$ & $1.07 \pm 0.50$ & $2.89 \pm 0.00$ \\
\hline $\mathrm{Cl}^{-}$ & $2.84 \pm 1.38$ & $15.7 \pm 0.00$ & $2.74 \pm 3.13$ & $10.0 \pm 1.81$ & $10.7 \pm 0.61$ & $4.81 \pm 2.86$ & $2.47 \pm 0.04$ & $0.04 \pm 0.00$ & $7.21 \pm 0.00$ & $10.7 \pm 2.43$ & ND & $0.04 \pm 0.00$ & ND & ND & ND \\
\hline $\mathrm{Na}^{+}$ & $2.51 \pm 2.55$ & $3.95 \pm 3.15$ & $2.69 \pm 2.23$ & $30.94 \pm 2.93$ & $3.55 \pm 0.40$ & $3.37 \pm 0.17$ & $1.12 \pm 1.06$ & $0.79 \pm 0.33$ & $7.00 \pm 0.00$ & $7.50 \pm 9.48$ & $1.32 \pm 0.58$ & $1.94 \pm 0.34$ & $1.78 \pm 0.25$ & $2.28 \pm 1.04$ & $3.30 \pm 0.00$ \\
\hline $\mathrm{V}$ & $0.23 \pm 0.07$ & $0.76 \pm 0.04$ & ND & ND & $0.51 \pm 0.03$ & ND & ND & $0.03 \pm 0.00$ & ND & $1.17 \pm 0.06$ & $1.30 \pm 0.58$ & $1.62 \pm 1.31$ & $0.65 \pm 0.35$ & $1.07 \pm 0.50$ & $2.89 \pm 0.14$ \\
\hline $\mathrm{Cr}$ & $0.08 \pm 0.06$ & $0.71 \pm 0.04$ & ND & $0.19 \pm 0.13$ & $0.13 \pm 0.01$ & $0.08 \pm 0.00$ & $0.24 \pm 0.33$ & $0.01 \pm 0.02$ & ND & $0.17 \pm 0.01$ & $0.07 \pm 0.00$ & $0.08 \pm 0.07$ & $0.31 \pm 0.35$ & $0.17 \pm 0.00$ & ND \\
\hline $\mathrm{Fe}$ & $0.11 \pm 0.11$ & $0.92 \pm 0.56$ & $0.02 \pm 0.02$ & $20.04 \pm 0.00$ & $0.04 \pm 0.00$ & $0.12 \pm 0.06$ & $0.20 \pm 0.28$ & $0.02 \pm 0.01$ & $0.01 \pm 0.00$ & $0.13 \pm 0.01$ & $0.03 \pm 0.01$ & $0.03 \pm 0.00$ & $0.02 \pm 0.02$ & $0.05 \pm 0.03$ & ND \\
\hline $\mathrm{Ni}$ & $0.07 \pm 0.00$ & $1.44 \pm 1.17$ & $0.01 \pm 0.00$ & $0.15 \pm 0.01$ & $0.24 \pm 0.01$ & ND & $0.03 \pm 0.02$ & $0.03 \pm 0.02$ & ND & $0.10 \pm 0.01$ & $0.04 \pm 0.03$ & $0.01 \pm 0.01$ & $0.04 \pm 0.00$ & $0.02 \pm 0.02$ & $0.06 \pm 0.00$ \\
\hline As & $0.36 \pm 0.02$ & $7.34 \pm 0.37$ & $0.39 \pm 0.06$ & $50.37 \pm 0.20$ & $0.48 \pm 0.02$ & $0.88 \pm 0.04$ & $0.21 \pm 0.30$ & $0.06 \pm 0.08$ & $0.13 \pm 0.01$ & $0.24 \pm 0.01$ & $0.00 \pm 0.00$ & $0.01 \pm 0.01$ & $0.02 \pm 0.00$ & $0.00 \pm 0.00$ & ND \\
\hline $\mathrm{Cd}$ & $0.22 \pm 0.01$ & $3.91 \pm 0.20$ & $0.14 \pm 0.01$ & $11.35 \pm 0.82$ & $3.46 \pm 0.17$ & $0.14 \pm 0.17$ & $0.31 \pm 0.19$ & $0.05 \pm 0.00$ & $0.03 \pm 0.01$ & $3.18 \pm 0.16$ & $0.00 \pm 0.00$ & $0.03 \pm 0.00$ & $0.04 \pm 0.04$ & $0.32 \pm 0.28$ & $0.54 \pm 0.03$ \\
\hline $\mathrm{Tb}$ & $1.20 \pm 0.17$ & $0.47 \pm 0.02$ & $0.48 \pm 0.07$ & $6.78 \pm 1.18$ & $2.32 \pm 0.12$ & $3.51 \pm 3.61$ & $0.36 \pm 0.04$ & $0.48 \pm 0.02$ & $0.80 \pm 0.04$ & $1.54 \pm 0.08$ & $0.12 \pm 0.10$ & $0.30 \pm 0.18$ & $0.05 \pm 0.36$ & $0.76 \pm 0.61$ & $1.30 \pm 0.07$ \\
\hline Er & $0.89 \pm 0.90$ & $26.9 \pm 20.9$ & $0.72 \pm 0.84$ & $4.81 \pm 4.56$ & $1.25 \pm 0.06$ & $1.77 \pm 1.95$ & $0.34 \pm 0.02$ & $0.26 \pm 0.30$ & $0.28 \pm 0.01$ & $3.51 \pm 0.18$ & $0.24 \pm 0.07$ & $0.23 \pm 0.21$ & $0.28 \pm 0.12$ & $0.81 \pm 0.61$ & $0.62 \pm 0.03$ \\
\hline $\mathrm{Yb}$ & $0.45 \pm 0.59$ & $16.7 \pm 0.84$ & $1.69 \pm 0.08$ & ND & $0.04 \pm 0.00$ & $2.73 \pm 1.91$ & ND & $0.19 \pm 0.23$ & $0.42 \pm 0.13$ & $2.77 \pm 0.14$ & $0.35 \pm 0.21$ & $0.16 \pm 0.14$ & $0.32 \pm 0.29$ & $0.44 \pm 0.28$ & $2.26 \pm 091$ \\
\hline $\mathrm{Lu}$ & $1.32 \pm 0.94$ & $11.8 \pm 0.59$ & $0.28 \pm 0.01$ & $12.53 \pm 0.13$ & $0.25 \pm 0.01$ & $0.83 \pm 0.82$ & $0.32 \pm 0.02$ & $0.18 \pm 0.05$ & $0.89 \pm 0.40$ & $2.68 \pm 0.13$ & $1.11 \pm 1.09$ & $0.34 \pm 0.51$ & $0.34 \pm 0.13$ & $0.49 \pm 0.44$ & ND \\
\hline
\end{tabular}

ND, Not Detected; 
1 Table S7 Actual navigation time of test vessels in different operating modes and their 2 proportion factors to the whole voyage

\begin{tabular}{cccc}
\hline Vessel ID & Operating mode & $\begin{array}{c}\text { Average daily } \\
\text { navigation time }(\mathrm{h})\end{array}$ & Proportion factor \\
\hline \multirow{4}{*}{$\mathrm{HH}$} & High speed & 1 & 0.08 \\
& Medium speed & 8 & 0.67 \\
& Low speed & 0.6 & 0.05 \\
& Idling & 1.6 & 0.13 \\
& Acceleration process & 0.4 & 0.03 \\
& Moderating process & 0.4 & 0.03 \\
\hline \multirow{3}{*}{ DFH } & Cruise & 16 & 0.67 \\
& Idling & 2 & 0.08 \\
& Acceleration process & 4 & 0.17 \\
& Moderating process & 2 & 0.08 \\
\hline \multirow{3}{*}{ XYH } & High speed & 10 & 0.63 \\
& Low speed & 2.5 & 0.16 \\
& Idling & 1.25 & 0.08 \\
& Acceleration process & 1 & 0.06 \\
& Moderating process & 1.25 & 0.08 \\
\hline
\end{tabular}

3 